\title{
Clinical and pathological predictors of the response to neoadjuvant anthracycline chemotherapy in locally advanced breast cancer
}

\author{
Mohamed M. Elmessiry, ${ }^{a} M D ;$ Maher M. Elzeiny, ${ }^{a} M D$; \\ Tarek A. Elfayomy, ${ }^{a} M D$; Haytham M. Fayed, ${ }^{a} M D$; \\ Basma Elsabaa, ${ }^{b} M D$; Mohamed Farouk, ${ }^{c} M D$
}

a) Department of Surgery, Alexandria University, Alexandria.

b) Department of Pathology, Alexandria University, Alexandria.

c) Department of Medical Oncology, Alexandria University, Alexandria.

\begin{abstract}
Purpose: The aim of this study is to determine clinical and histopathological characteristics that predict tumor response to anthracycline-based neoadjuvant chemotherapy in operable locally advanced breast cancer.

Patients and methods: We studied primary tumor core tissue biopsies from 60 patients with operable locally advanced breast cancer. Patients received anthracycline-based chemotherapy. The World Health Organization (WHO) criteria were used for staging and the Nottingham modification of Bloom \& Richardson scoring system was used for tumor grading. Immunohistochemical staining for ER, PgR, HER-2 and Ki-67 was performed. Clinical, histopathological and immunohistochemical characteristics were analyzed and correlated with pathological response to neoadjuvanty chemotherapy.

Results: Mean patients' age was 50.8 99.2 years. Sixty five percent of patients were postmenopausal. Ninety percent had infiltrating ductal carcinoma; NOS. Five percent, 25\% and $70 \%$ of patients had stage IIB, IIIA and IIIB disease respectively. Fifty five percent of tumors were high grade. Sixty five percent of cases received neoadjuvant chemotherapy (NCT) in the form of FAC while 15\% received FEC (doxorubicin was replaced with epirubicin) and $20 \%$ received AC (without 5-flurouracil). Disease progression was recorded in $15 \%$ of patients while stable disease was observed in $25 \%$ of patients and $55 \%$ recorded partial response. Only $5 \%$ of patients concurred complete response. Breast conservative surgery $(B C S)$ was conducted in $33.3 \%$ of responders to NCT ( $p$ 0.00). Factors associated with a better tumor response were younger age ( $p 0.03)$, smaller tumor size ( $p 0.01)$, lower nodal stage ( $p 0.00)$, high pathological grade (p 0.025), high Ki-67 labeling index, ( $p 0.00)$ and low expression of ER, PgR and Her2 ( $p$ 0.00). There was no significant relation between tumor response and each of menopausal status, tumor stage, histological type, presence of lymphovascular space invasion (LVSI) or serum levels of tumor markers [CA15.3 \& CEA].

Conclusion: Operable, locally advanced breast cancer with large tumor size, higher nodal stage, positive immunohistochemical reaction to ER, $P g R$ \& Her2, lower Ki-67 labeling index and a lower tumor grade predict a lower response to anthracycline-based NCT. These patients could alternatively benefit from radical resection or a different line of NCT to obtain a better response with the possibility of $B C S$.
\end{abstract}

Key words: Neoadjuvant chemotherapy; operable locally advanced breast cancer; pathological response; clinical and pathological predictors. 


\section{Introduction:}

Locally advanced breast cancer (LABC) constitutes a heterogeneous group of tumors of varying clinical presentations and biological behavior. It represents up to 10$20 \%$ of newly diagnosed breast cancers in western countries. ${ }^{1}$ In developing countries, it represents up to 50-60\% due to lack of screening programs and decreased public awareness of the importance of early detection of breast cancer. ${ }^{1}$ The clinical management of LABC is complex and should be tailored to the individual patient either by neoadjuvant or adjuvant chemotherapy plus surgery. Some patients with LABC have potentially operable tumors including stages IIB, IIIA and some cases of stage IIIB. Some advantages of the administration of NCT in operable breast cancer include possible determination of tumor response in vivo, earlier treatment of micrometastases, diminishing drug resistance, and increasing the possibility of breast conservation. ${ }^{2,3}$ In addition, some clinical studies have shown that responders to NCT have better diseasefree survival rates. ${ }^{4}$ Generally, there is wider experience with the use of anthracyclines in the treatment of these patients. However; there is a significant variability in the pathologic response to anthracycline-based NCT, with approximately $65-85 \%$ overall response rate, $5-15 \%$ complete response and $15-35 \%$ stable or progressive disease. ${ }^{5-10}$ Currently, the underlying mechanism for this variability is unclear. The identification of clinical and histopathological parameters that accurately predict such response to treatment is valuable in optimizing NCT so that nonresponders could be offered alternative and more effective chemotherapy or radical surgery. Previous studies have focused on the correlation between tumor response to chemotherapy and diverse factors, such as histological grade, DNA ploidy, cell kinetics, and estrogen receptor status of the primary tumor. However, most of these studies have unfortunately yielded confounding results. ${ }^{11-17}$

\section{Purpose:}

The aim of this study was to determine clinicopathological features that can predict response of operable locally advanced breast cancer to NCT.

\section{Patients and methods:}

Sixty patients with operable LABC (stages IIB, IIIA, or IIIB as defined by the American Joint Committee on Cancer Classification "AJCC" System) were studied during the period between March 2009 and March 2012. All patients underwent tumor core tissue biopsy to confirm the presence of invasive carcinoma and to evaluate the primary tumor pathological and immunohistological features. Patients with preoperative chemo or radiotherapy, ductal carcinoma in-situ with minor invasive component were excluded. Prior to every treatment cycle, radiological three-dimensional measurements of the tumor were performed. Then, all patients received neoadjuvant anthracyclinebased chemotherapy administrated at 21day-intervals. Neoadjuvant regimens consisted of $600 \mathrm{mg} / \mathrm{m}^{2}$ 5-fluorouracil, $60 \mathrm{mg} / \mathrm{m}^{2}$ doxorubicin, and $600 \mathrm{mg} / \mathrm{m}^{2}$ cyclophosphamide (FAC) or $600 \mathrm{mg} / \mathrm{m}^{2}$ 5-fluorouracil, $60 \mathrm{mg} / \mathrm{m}^{2}$ epirrubicin, and $600 \mathrm{mg} / \mathrm{m}^{2}$ cyclophosphamide (FEC) or $60 \mathrm{mg} / \mathrm{m}^{2}$ doxorubicin and $600 \mathrm{mg} / \mathrm{m} 2$ cyclophosphamide (AC).

After achieving the maximum response (defined as no more reduction in tumor size for 2 successive cycles) after a minimum of three to a maximum of six cycles of chemotherapy, patients and tumor responses were evaluated clinically and radiologically by mammography and ultrasonography of both breasts and axillae. Responders were offered breast conservative therapy or modified radical mastectomy (MRM) followed by the same chemotherapy regimen as an adjuvant treatment. Non-responders (if still resectable) were offered MRM followed by other lines of chemotherapy.

Clinical \& histopathological data were collected \& analyzed to assess possible correlations with tumor response to anthracycline-based NCT. Compiled data included clinical (patient's age, menopausal status, tumor location, size and stage), 
radiological (tumor and nodal staging), laboratory (serum levels of tumor markers namely CEA\& CA 15.3) and histopathological (histological type, grade, presence of lymphovascular LVSI, hormone receptor status (ER \& PgR), HER-2 expression, Ki-67 labeling index) in addition to therapeutic data (type and number of cycles of NCT, tumor and nodal response to NCT).

\section{Histological analysis:}

Sections of formalin-fixed, paraffin embedded tumor core biopsies stained with Hematoxylin \& Eosin (H\&E) were studied. Tumor type was determined as per the guidelines of the WHO classification criteria (2003). Based on the assessment of formation of tubules "1-3", nuclear atypia "13 ", and mitotic counts " $1-3$ " the appropriate histologic grade was assigned to the tumor using the criteria of Nottingham scoring system "Modified Bloom and Richardson" scoring system. ${ }^{15}$ Scores were added up to formulate a final score out of 9. A score of up to 5 determined a Grade 1 tumor, 6-7 a Grade 2 tumor and 8-9 Grade 3 tumor. Presence of LVSI \& lymphocytic infiltration were also assessed.

\section{Immunohistochemical studies:}

Immunohistochemical staining for estrogen receptors (ER), progesterone receptors (PgR), HER-2 and Ki-67 was performed on 3- $\mu$ m-thick sections of formalin-fixed paraffin-embedded tissue from core tissue biopsies mounted onto positively charged slides. Slides were de-waxed and then re-hydrated. Following microwave pretreatment, monoclonal antibodies to ER, PgR, HER-2, and Ki-67 were applied overnight at $4^{\circ} \mathrm{C}$. Then incubation with peroxidase labeled secondary antibody and chromogen was conducted. Each section was examined at low magnification (100x) to identify percentage of positive staining cells and staining intensity. Negative and positive control slides were included in each run. Staining for ER, PgR and Ki67 was always nuclear while for HER-2 it was membranous. For ER and PgR semiquantitative scoring, the Allred scoring system ${ }^{18}$ which is the sum of a score for percentage of positively staining cells (no staining $=0$, staining in $<1 \%$ of cells $=1,1$ to $10 \%=2,10$ to $33 \%=3,33$ to $67 \%$ $=4$ and $67 \%$ to $100 \%=5$ ) and a score for intensity (absent $=0$, weak $=1$, moderate $=$ 2 , strong $=3$ ). A $0-2$ score was considered negative. Allred scores 3-4 were considered $1+, 5$ and 6 were considered $2+, 7$ and 8 were considered 3+. HER2 was graded according to ASCO/CAP guidelines ${ }^{19}$ as negative when there was no immunostaining or when there was weak immunostaining of less than $30 \%$ $(1+)$, equivocal $(2+)$ when there was complete uniform or weak membranous staining and positive (3+) when there was uniform intense membranous staining in at least $30 \%$ of cells. Proliferative activity was assessed in terms of Ki67 labeling index as either less than 20\% (low) or $>20 \%$ (high). ${ }^{20}$

\section{Assessment of response:}

Clinical and radiological changes in preoperative tumor size after conclusion of chemotherapy were recorded. A complete response was considered when no residual tumor was detected clinically or radiologically; a partial response was defined as tumor reduction to less than $50 \%$ of the baseline size; stable disease defined as less than $50 \%$ reduction in tumor size or less than $25 \%$ increase in tumor size. Progressive disease was defined as more than $25 \%$.increase in tumor size. ${ }^{21}$ Pathologically, specimens with no residual viable tumor cells were judged to have complete pathological response (pCR), presence of one or more foci of malignant viable cells measuring less than 1 millimeter was judged as near complete (microscopic) response while a residual tumor larger than 1 millimeter was judged as partial response. ${ }^{22}$

\section{Statistical analysis:}

Chi-square and ANOVA tests were used to compare categorical and continuous variables, respectively.

\section{Results:}

Pretreatment evaluation showed that: the mean age was $50.8 \pm 9.2$ years. Sixty five percent of patients were postmenopausal. The mean length of largest tumor dimensions was 
$7.5 \pm 2.4 \mathrm{~cm}$. Tumor stages ranged between T3 (18\%), T4b (72\%) and T4d (10\%). N1 nodal status was recorded in $55 \%$ of cases and $\mathrm{N} 2$ in 35\%. The remaining $10 \%$ were $\mathrm{N} 0$. Regarding clinical stage, 5\%, 25\% and 70\% of patients recorded stages IIB, IIIA and IIIB respectively. Histopathological evaluation revealed infiltrating ductal carcinoma; NOS in $90 \%$ of cases and infiltrating lobular carcinoma in the rest. Fifty five percent of cases were grade III. LVSI was detected in $85 \%$. ER/PgR hormone receptor positivity was detected in $40 \%$. Her- 2 overexpression was reported in $20 \%$ of cases while $38.3 \%$ of tumors showed high proliferative activity (high Ki-67 labeling index).

Sixty five percent of the patients received NCT in the form of FAC including $600 \mathrm{mg} /$ $\mathrm{m} 25$-fluorouracil, $60 \mathrm{mg} / \mathrm{m}^{2}$ doxorubicin, and $600 \mathrm{mg} / \mathrm{m}^{2}$ cyclophosphamide. $15 \%$ received FEC (doxorubicin replaced with epirubicin) and $20 \%$ received AC (without 5-flurouracil).

The overall clinical response rate was $60 \%$. Fifty three percent of patients had partial response while $6.7 \%$ had a complete response. Stable disease was observed in $25 \%$ of patients and $15 \%$ of patients developed disease progression. Histopathological assessment revealed complete pathological response in $5 \%$ of cases, near complete pathological response in $1.7 \%$ and partial pathological response in $53.3 \%$. Breast conservative surgery (BCS) was performed in $33.3 \%$ of responders to NCT (p 0.00).

Factors associated with a better tumor response to NCT were younger age ( $\mathrm{p} 0.03$ ), smaller tumor size (p 0.01), lower nodal stage (p 0.00), higher tumor grade ( $\mathrm{p} 0.025)$, higher Ki67 labeling index (p 0.00), negative ER/ PgR status, lack of Her2 over-expression ( $\mathrm{p}$ 0.00), elevated serum CA15.3 (p 0.00) and higher number of NCT cycles ( $\mathrm{p} 0.00$ ). There was no significant relation between response to treatment and any of menopausal status, $\mathrm{T}$ stage, histological type, LVSI or serum CEA level.

In the multivariate analysis, patient's age $(\mathrm{p}=0.001)$, tumor grade $(\mathrm{P}=0.003)$ and Ki67 labeling index $(\mathrm{P}=0.001)$ were the only independent predictive factors of response to anthracycline-based NCT.

Using logistic regression analysis of different combinations of predictive variables revealed that the combination of young age, small tumor size and negative nodal status is the strongest predictor of tumor response to NCT $\left(x^{2} 45.778, p<0.000\right)$. The accuracy of this combination was $91.7 \%$ 


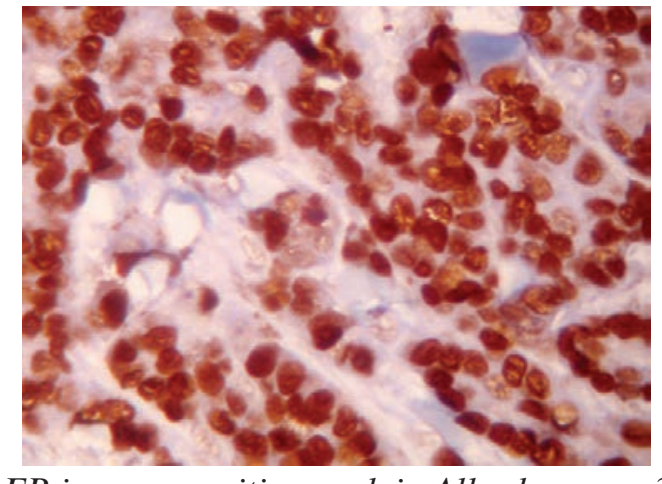

ER immunopositive nuclei, Allred score +3

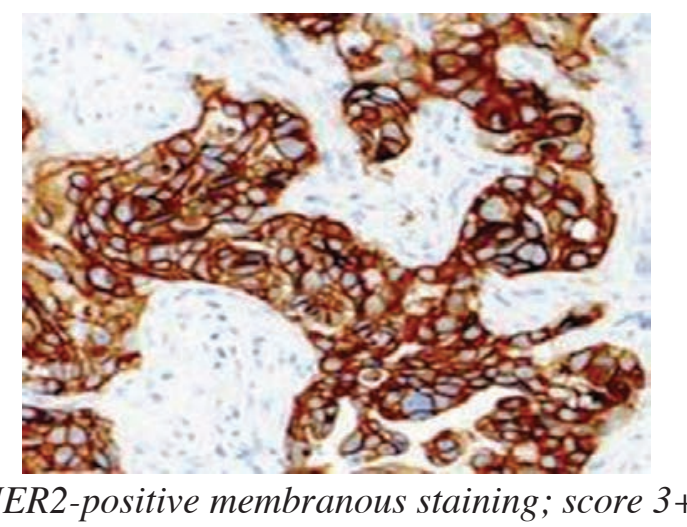

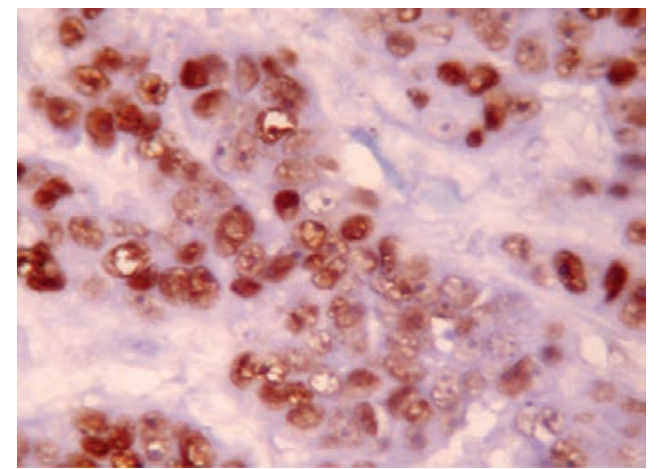

PgR immunopositive nuclei, Allred score 3+

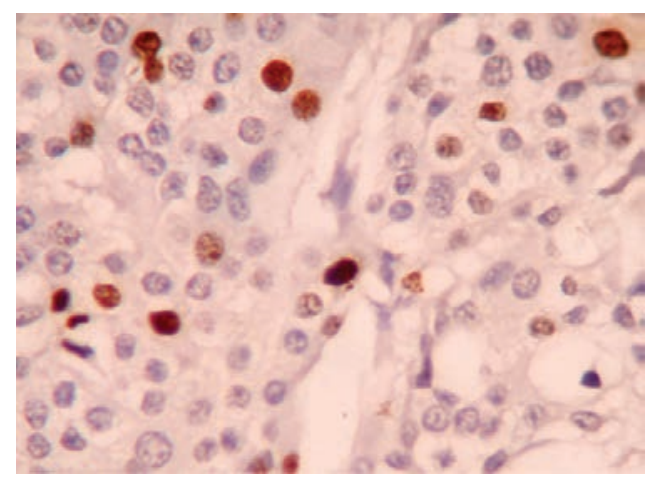

Ki-67 nuclear staining in, breast carcinoma

Figure (1): Duct to mucosa PJ: Posterior outer row is completeright angle in the pancreatic duct.

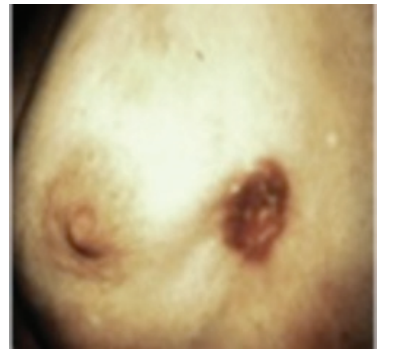

Before CT

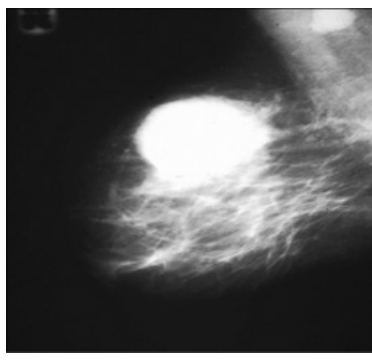

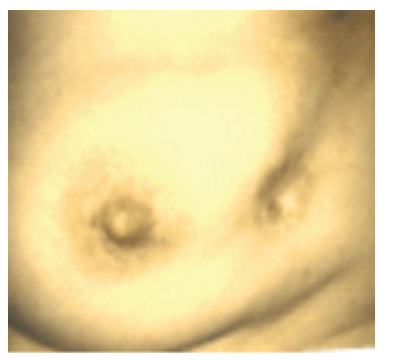

After CT

Figure (2): A case with LABC showing partial response after neoadjuvant CT.
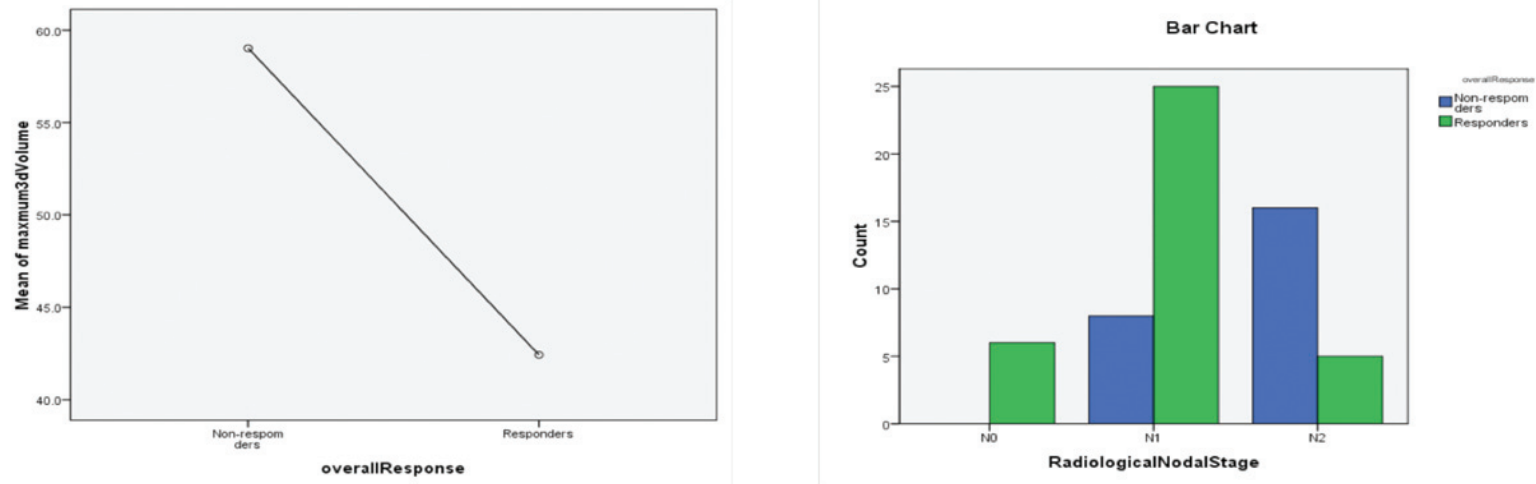

Figure (3): Relation between response to neoadjuvant CT and tumor size \& nodal stage. 
Table (1): Relation between clinical, radiological \& laboratory findings and tumor response to $N C T$.

\begin{tabular}{|c|c|c|c|c|}
\hline & & Non-responders & Responders & $\mathrm{P}$ \\
\hline \multirow[t]{2}{*}{ Age } & Mean & 53 & 48 & \multirow{2}{*}{$0.03^{*}$} \\
\hline & $\mathrm{SD}$ & 8.5 & 9.5 & \\
\hline \multirow[t]{2}{*}{ Menopausal status } & premenopausal & 6 & 15 & \multirow{2}{*}{0.147} \\
\hline & postmenopausal & 18 & 21 & \\
\hline \multirow[t]{2}{*}{ Tumor largest diameter } & Mean & 8.9 & 6.5 & \multirow{2}{*}{$0.008 *$} \\
\hline & SD & 2.6 & 1.8 & \\
\hline 3D size & Mean & 59 & 42.4 & \multirow{2}{*}{$0.002 *$} \\
\hline SD & & 21.9 & 12.3 & \\
\hline \multirow[t]{3}{*}{ T stage } & $\mathrm{T} 3$ & 3 & 8 & \multirow{3}{*}{0.58} \\
\hline & $\mathrm{T} 4 \mathrm{~b}$ & 18 & 25 & \\
\hline & $\mathrm{T} 4 \mathrm{~d}$ & 3 & 3 & \\
\hline \multirow[t]{3}{*}{$\mathrm{N}$ status } & No & 0 & 6 & \multirow{3}{*}{$0.000 *$} \\
\hline & N1 & 8 & 25 & \\
\hline & $\mathrm{N} 2$ & 16 & 5 & \\
\hline \multirow[t]{3}{*}{ Clinical stage } & IIB & 0 & 3 & \multirow{3}{*}{0.34} \\
\hline & IIIA & 6 & 9 & \\
\hline & IIIB & 18 & 24 & \\
\hline CEA & Mean & 11.2 & 11.9 & \multirow{2}{*}{0.872} \\
\hline $\mathrm{SD}$ & & 13.5 & 20.2 & \\
\hline \multirow[t]{2}{*}{ CA 15.3} & Mean & 18 & 30.6 & \multirow{2}{*}{$0.001 *$} \\
\hline & SD & 11.5 & 13.2 & \\
\hline
\end{tabular}


Table (2): Relation between histopathological features and tumor response to NCT.

\begin{tabular}{|c|c|c|c|c|}
\hline & & Non-responders & Responders & $\mathrm{P}$ \\
\hline \multirow[t]{2}{*}{ Histological type } & IDC & 22 & 32 & \multirow{2}{*}{0.72} \\
\hline & ILC & 2 & 4 & \\
\hline \multirow[t]{2}{*}{ Tumor grade } & Grade II & 19 & 7 & \multirow{2}{*}{$0.000 *$} \\
\hline & Grade III & 5 & 29 & \\
\hline \multirow[t]{2}{*}{ LVSI } & Absent & 3 & 6 & \multirow{2}{*}{0.478} \\
\hline & Present & 21 & 30 & \\
\hline \multirow[t]{3}{*}{ ER score } & Negative & 6 & 30 & \multirow{3}{*}{$0.000 *$} \\
\hline & $1+/ 2+$ & 3 & 3 & \\
\hline & $3+$ & 15 & 3 & \\
\hline \multirow[t]{3}{*}{$\operatorname{PgR}$} & Negative & 10 & 21 & \multirow{3}{*}{$0.022 *$} \\
\hline & $1+/ 2+$ & 4 & 11 & \\
\hline & $3+$ & 10 & 4 & \\
\hline \multirow[t]{3}{*}{ HER 2} & $0 /+1$ & 9 & 21 & \multirow{3}{*}{$0.018 *$} \\
\hline & +2 & 3 & 15 & \\
\hline & +3 & 12 & 0 & \\
\hline \multirow[t]{2}{*}{ Ki-67 } & Low $(<20 \%)$ & 12 & 3 & \multirow{2}{*}{$0.000^{*}$} \\
\hline & $\begin{array}{l}\text { Medium + high } \\
(>20 \%)\end{array}$ & 12 & 33 & \\
\hline
\end{tabular}

Table (3): Relation between tumor response to NCT and regimen \& number of cycles of CT.

\begin{tabular}{|l|l|c|c|c|}
\hline \multirow{3}{*}{ Type of CT } & & Non-responders & Responders & P \\
\cline { 2 - 4 } & FAC & 12 & 27 & \\
\cline { 2 - 4 } & FEC & 6 & 3 & \multirow{2}{*}{0.103} \\
\cline { 2 - 4 } No. of cycles & AC & 6 & 6 & \\
\cline { 2 - 4 } & 3 & 9 & 0 & \multirow{2}{*}{$0.001^{*}$} \\
\cline { 2 - 4 } & 4 & 3 & 9 & \\
\cline { 2 - 4 } & 5 & 3 & 12 & \\
\hline
\end{tabular}


Table (3): Clinical and biological parameters in relation to their predictive value for achieving a partial/complete response to NCT: multivariate analysis.

\begin{tabular}{|l|c|}
\hline Biologic parameters & P value \\
\hline Age & $0.001^{*}$ \\
\hline Tumor largest diameter & $0.000^{*}$ \\
\hline N status & $0.002^{*}$ \\
\hline CA 15.3 level & 0.66 \\
\hline Tumor grade & $0.003^{*}$ \\
\hline ER score & 0.07 \\
\hline PgR score & 0.09 \\
\hline HER2 score & 0.18 \\
\hline Ki67 level & $0.002^{*}$ \\
\hline
\end{tabular}

\section{Discussion:}

$60 \%$ of patients in this study presented a clinical response to anthracycline-based neoadjuvant chemotherapy. $53.3 \%$ had pCR and only $6.7 \%$ of patients had a cCR. In relation to the pathological response, $55 \%$ had pPR and only $5 \%$ of patients had a cPR. It has been published in the literature that the response to induction chemotherapy varies between $3 \%$ and $30 \%$ for cCR and 30-80\% for pCR.2,5-11 Breast conservative surgery (BCS) was possible in $20 \%$ of patients after downstaging. It is published in the literature that the rate of breast conservation after induction chemotherapy varies between $15 \%$ and $45 \% .5-9$

We found that a smaller tumor size and few metastatic nodes at diagnosis were related to a better partial and complete response. This could be due to the fact that bigger tumors could have extended zones of necrosis and presence of intratumoral edema that increases interstitial pressure. All these features make it difficult for the chemotherapy to penetrate into the tumor. Moreover, a larger size means that more cells have been duplicating, favoring more mutations and resistance to chemotherapy.

A strong association between clinical and pathological response with tumor grade was found; this correlation has been repeated in other studies. ${ }^{23-27}$ Nevertheless, there are isolated reports that suggest the opposite. ${ }^{14}$
Our study showed a significant difference in response to neoadjuvant chemotherapy among patients with high proliferating activity when compared to patients with tumors with a low proliferative activity demonstrated by a high Ki-67 labeling index. Several studies have evaluated cell proliferation and concluded a significant correlation between high proliferative activity and a greater sensitivity to chemotherapy. ${ }^{14,26,28}$

In our study, there was an association between HER2 overexpression and tumor response to NCT. Nevertheless, correlation between HER2 overexpression and response to chemotherapy is controversial. The Cancer Leukemia Group B B8451 Study found that patients who received high doses of doxorubicin with overexpression of HER2 showed a benefit in survival. ${ }^{25}$ In other studies, Overexpression of HER2, defined as $3+$ IHC staining did not influence the clinical or pathological response to anthracyclinebased chemotherapy. ${ }^{30-32}$

In our study, ER-negative status was a predictor of clinical and pathological response to neoadjuvant chemotherapy. Previous studies demonstrated that tumors with positive ER had a worse pathological response to NCT compared to negative ER. ${ }^{9,28}$ Colleoni found a significantly higher clinical response rate in negative ER and PgR tumors, compared to those where ER and/or PgR were expressed. ${ }^{33}$ These results could be 
explained, in part, by the relation that exists between absence of these receptors and a higher tumor grade, being this last one an important response factor.

We found a greater response to treatment with doxorubicin compared with epirubicin. The number of cycles received also influenced the clinical response. It is well known that with a greater number of cycles, it is more likely to achieve a complete clinical response.

In conclusion, we found that patients with locally advanced breast cancer tumors with a large volume, advanced node status (N2), low cell proliferation rate, and lower differentiation grade according to the SBR score have a lower response to anthracyclinebased NCT. Patients with tumors with these characteristics could be candidates for radical resection or a different line of neoadjuvant $\mathrm{CT}$ to obtain a better response with the possibility of BCS.

\section{References:}

1- Rustogi A, Budrukkar A, Dinshaw K, Jalali $\mathrm{R}$ : Management of locally advanced breast cancer: Evolution and current practice. $J$ Can Res Ther 2005; 1: 21-30.

2- Wolff A, Davidson N: Primary systemic therapy in operable breast cancer. $J$ Clin Oncol 2000; 18: 1558-1569.

3- Hortobagyi G: Treatment of breast cancer. N Engl J Med 1998; 339: 974-984.

4- Aapro M: Neoadjuvant therapy in breast cancer: Can we define its role? The Oncologist 2001; 6: 366-369.

5- Van der Hage JA, van de Velde CJH, Julien JP, et al: Preoperative chemotherapy in primary operable breast cancer: Results from the European Organization for Research and Treatment of Cancer trial 10902. J Clin Oncol 2001; 19: 4224-4237.

6- Makris A, Powles TJ, Ashley SE, et al: A reduction in the requirements for mastectomy in a randomized trial of neoadjuvant chemoendocrine therapy in primary breast cancer. Ann Oncol 1998; 9: 1179-1184.

7- Smith IC, Heys SD, Hutcheon AW, et al: Neoadjuvant chemotherapy in breast cancer: Significantly enhanced response with docetaxel. J Clin Oncol 2002; 20: 1456-1466.

8- Schwartz GF, Birchansky CA, Komarnicky LT, et al: Induction chemotherapy followed by breast conservation for locally advanced carcinoma of the breast. Cancer 1994; 73: 362-369.

9- Fisher B, Bryant J, Wolmark N, et al: Effect of preoperative chemotherapy on the outcome of women with operable breast cancer. J Clin Oncol 1998; 16: 2672-2685.

10-Kuerer HM, Newman LA, Smith TL, et al: Clinical course of breast cancer patients with complete pathologic primary tumor and axillary lymph node response to doxorubicin- based neoadjuvant chemotherapy. J Clin Oncol 1999; 17: 460-469.

11-Ring AE, et al: Oestrogen receptor status, pathological complete response and prognosis in patients receiving neoadjuvant chemotherapy for early breast cancer. $\mathrm{Br} \mathrm{J}$ Cancer 2004; 91: 2012-2017.

12-Masters JR, Camplejohn RS, Millis RR, Rubens RD: Histologic grade, elastosis, DNA ploidy and response to chemotherapy. Br J Cancer 1987; 55: 455-457.

13-Aas T, et al: Primary systemic treatment with weekly doxorubicin monotherapy in women with locally advanced breast cancer: Clinical experience and parameters predicting outcome. Acta Oncol 1996; 35: 5-8.

14-Sjostrom J, et al: A multivariate analysis of tumor biologic factors predicting response to cytotoxic treatment in advanced breast cancer. Br J Cancer 1998; 78: 812-815.

15-Elston CW, Ellis IO: Assessment of histological grade. In: The breast. Elston CW and Ellis IO (Editors); Edinnburgh; New York: Churchill Livigstone (Publisher); 1998; Vol 13; p. 356-84.

16-Collecchi P, et al: Primary chemotherapy in locally advanced breast cancer (LABC): Effects on tumor proliferative activity, bcl2 expression and the relationship between tumor regression and biological markers. Eur J Cancer 1998; 34: 1701-1704.

17-Ozmen V, et al: Biological considerations in locally advanced breast cancer treated 
with anthracycline-based neoadjuvant chemotherapy: thymidine labelling index is an independent indicator of clinical outcome. Breast Cancer Res Treat 2001; 68: 147-157.

18-Collins LC, Botero ML, Schnitt SJ: Bimodal frequency distribution of estrogen receptor immunohistochemical staining results in breast cancer: An analysis of 825 cases. Am J Clin- Pathol 2005; 123: 16-20.

19-Allen M Gown: Current issues in ER and HER2 testing by IHC in breast cancer. Modern Pathology 2008; 21: 8-15.

20-DeCensi A, Guerrieri-Gonzaga A, Gandini S, et al: Prognostic significance of Ki-67 labeling index after short-term presurgical tamoxifen in women with ERpositive breast cancer. Ann Oncol 2011; 22(3): 582-587.

21-PetitT, WiltM, Velten M, etal:Comparative value of tumour grade, hormonal receptors, Ki-67, HER-2 and topoisomerase II alpha status as predictive markers in breast cancer patients treated with neoadjuvant anthracycline-based chemotherapy. Eur $J$ Cancer 2004; 40: 205-211.

22-Candelaria M, Chanona-Vilchis J, Cetina $\mathrm{L}$, et al: Prognostic significance of pathological response after neoadjuvant cchemotherapy or chemoradiation for locally advanced cervical carcinoma. Int Semin Surg Oncol 2006; 3-13.

23-Prisacki HB, Karramani C, Modlichi $\mathrm{O}$, et al: Predictive biological markers for response of invasive breast cancer to anthracycline - based primary chemotherapy. Anticancer research 2005; 25: 4615-4622.

24-Wang J, et al: Assessment of histologic features and expression of biomarkers in predicting pathologic response to anthracycline-based neoadjuvant chemotherapy in patients with breast carcinoma. Cancer 2002; 94: 3107-3114.
25-Vincent-Salomon A, et al: ERBB2 overexpression in breast carcinomas: no positive correlation with complete pathological response to preoperative highdose anthracycline-based chemotherapy. Eur J Cancer 2000; 36: 586-591.

26-Page DL, et al: Prediction of nodenegative breast cancer outcome by histologic grading and S-phase analysis by flow cytometry: An Eastern Cooperative Oncology Group Study (2192). Am J Clin Oncol 2001; 24: 10-18.

27-Stearns V, et al: A prospective randomized pilot study to evaluate predictors of response in serial core biopsies to single agent neoadjuvant doxorubicin or paclitaxel for patients with locally advanced breast cancer. Clin Cancer Res 2003; 9: 124-133.

28-Thor AD, et al: ErbB-2, p53 and efficacy of adjuvant therapy in lymph node-positive breast cancer. J Natl Cancer Inst 1998; 90 : 1346-1360.

29-Pegram MD, et al: The effect of HER-2/ neu overexpression on chemotherapeutic drug sensitivity in human breast and ovaian cancer cells. Oncogene 1997; 15: 537-547.

30-Zhang F, et al: Correlation between HER2 expression and response to neoadjuvant chemotherapy with 5-fluorouracil, doxorubicin, and cyclophosphamide in patients with breast carcinoma. Cancer 2003; 97: 1758-65.

31-Pu RT, Schott AF, Sturtz DE, Griffith KA, Kleer CG: Pathologic features of breast cancer associated with complete response to neoadjuvant chemotherapy: Importance of tumor necrosis. Am J Surg Pathol 2005; 29: 354-358.

32-Colleoni M, et al: Response to primary chemotherapy in breast cancer patients with tumors not expressing estrogen and progesterone receptors. Ann Oncol 2000; 11: 1057-1059. 